\title{
Efficacy of Manual Acupuncture Versus Placebo Acupuncture for Generalized Anxiety Disorder (Gad) in Perimenopause Women : Study Protocol for a Randomized Controlled Trial
}

Xin Liu

Guangzhou University of Chinese Medicine

Xiaoyan Xie

Guangzhou University of Chinese Medicine

Yingjia Li

Guangzhou University of Chinese Medicine

Meichen Li

Guangzhou University of Chinese Medicine

\section{Yuting Wang}

Guangzhou University of Chinese Medicine

Nanbu Wang

Guangzhou University of Traditional Chinese Medicine First Affiliated Hospital

Muxi Liao

Guangzhou University of Traditional Chinese Medicine First Affiliated Hospital

Lixing Zhuang ( $\nabla$ zhuanglixing@163.com )

Guangzhou University of Traditional Chinese Medicine First Affiliated Hospital https://orcid.org/00000001-7276-9511

\section{Research Article}

Keywords: generalized anxiety disorder, acupuncture, protocol

Posted Date: August 18th, 2021

DOI: https://doi.org/10.21203/rs.3.rs-577572/v1

License: (c) (1) This work is licensed under a Creative Commons Attribution 4.0 International License.

Read Full License 
Version of Record: A version of this preprint was published at Trials on November 24th, 2021. See the published version at https://doi.org/10.1186/s13063-021-05756-x. 


\title{
Efficacy of manual acupuncture versus placebo acupuncture
}

\section{for generalized anxiety disorder (GAD) in perimenopause}

\section{women: study protocol for a randomized controlled trial}

Xin Liu, ${ }^{1}$ Xiaoyan Xie, ${ }^{1}$ Yingjia Li, ${ }^{1}$ Meichen Li, ${ }^{1}$ Yuting Wang, ${ }^{1}$ Nanbu Wang, ${ }^{2}$ Muxi Liao $^{2}$ and Lixing Zhuang ${ }^{2}$

Xin Liu and Xiaoyan Xie contributed equally to this work and should be considered as co-authors.

Correspondence: Lixing Zhuang, Department of Rehabilitation, The First Affiliated Hospital of Guangzhou University of Chinese Medicine, No.16 Airport Road, Baiyun district, Guangzhou, ChinaTel +86 13822287775

Email zhuanglixing@163.com

\begin{abstract}
Background: Generalized anxiety disorder (GAD) is common among perimenopause women. Drug treatment and cognitive behavioral therapy have their pros and cons. Acupuncture may be an effective treatment for GAD, but evidence is limited. The pathogenesis of GAD is not yet clear, but it is related to hypothalamic-pituitary-adrenal axis and its excretion, cortisol (CORT) and adrenocorticotropic hormone (ACTH). The object of this study is to assess the efficacy of manual acupuncture (MA) versus placebo acupuncture (PA) for perimenopause women with GAD.

Methods: This study is a single center, randomized, double blind clinical trial that will be conducted in the First Affiliated Hospital of Guangzhou University of Chinese Medicine. A total of 112 eligible GAD patients will be randomly assigned (1:1) to receive MA $(n=56)$ or PA $(n=56)$ three times per week for four weeks. The primary outcome measure will be the score of GAD-7. The secondary outcome measures will be the score of HAMA and PSQI, and the level of CORT and ACTH. The evaluation will execute at baseline, 2 weeks, the end of the treatment and a follow-up period. All main analyses will be carried out based on the intention-to-treat (ITT) principle.

Discussion: This study intends to conduct to compare the efficacy between MA and PA in the treatment of perimenopause woman with GAD, and to further study the mechanisms of effect.

Trial registration: Chinese Clinical Trial Registry, ID: ChiCTR2100046604.
\end{abstract} Registered on 22 May 2021. URL: http://www.chictr.org.cn.

Keywords: generalized anxiety disorder, acupuncture, protocol

\section{Background}

Generalized anxiety disorder (GAD) belongs to mental disorders, which including both mental and physical symptoms without pathological changes in brain ${ }^{[1]}$. 
GAD patients with general apprehensiveness or worry that is not restricted to any particular stimulus as the primary clinical feature ${ }^{[2]}$. According to an epidemical research of European, the incidence rate of GAD is $6.2 \%$ in the lifetime ${ }^{[3]}$. Moreover, female are 1.5 to 2 times more exposure to GAD than male ${ }^{[4]}$. To our knowledge, the prevalence of female aged between 45 -year-old to 54-year-old is the highest ${ }^{[5]}$. This phenomenon indicates that perimenopause women are more likely to suffer from GAD. As one of the first-line pharmacotherapy of $\mathrm{GAD}^{[6]}$, Serotonin reuptake inhibitors (SSRIs) inhibits the reuptake of serotonin to increase the concentration of serotonin in synaptic cleft to improve the level of serotonin in brain which is believed to attenuate anxious symptoms ${ }^{[7]}$. But patients' condition might aggravate in the first week when first taking the SSRIs ${ }^{[8]}$. So some of the patients refuse to take drugs because of the adverse events. They seek for the help of psychiatrists and some of them probably received cognitive behavioral therapy (CBT). Studies ${ }^{[9,10]}$ have shown that $\mathrm{CBT}$ is an effective treatment for GAD. However, due to the shortage of professionals and expensive treatments, CBT is not such beneficial for most patients $^{[11]}$. Therefore, patients turn to find the help from alternative therapies such as acupuncture.

Although the pathogenesis of GAD is not yet clear, the current studies believes that it is related to abnormal secretory function of the hypothalamic-pituitary-adrenal axis (HPA axis) ${ }^{[12]}$. Cortisol (CORT) synthesized by the adrenal cortex is one of the final products of the HPA axis. CORT and adrenocorticotropic hormone (ACTH) levels in GAD patients are higher than normal people ${ }^{[13,14]}$. The elevation of CORT and ACTH would be caught as a negative feedback by hypothalamus which will downregulate the stress response ${ }^{[15,16]}$. Therefore, the decline of CORT and ACTH levels can predict the degree of improvement that a treatment produces ${ }^{[17]}$.

Therefore, we designed a randomized controlled trial that focus on the efficacy of manual acupuncture versus placebo acupuncture on GAD in perimenopause women. To evaluate the therapeutic efficacy of GAD, the primary outcome measure used in our protocol is the change in scores of Generalized Anxiety Disorder Scale (GAD-7). The secondary outcome measures are the changes in scores of the Hamilton Anxiety Scale (HAMA) and Pittsburgh sleep quality index (PQSI), and the laboratory indicators related to the current possible pathogenesis of GAD, mainly including CORT and ACTH. This protocol will help explore the efficacy and possible mechanism of acupuncture on GAD.

\section{Methods}

\section{Study design}

This is a randomized controlled and patient-and-assessor-blind trial. A total of 112 participants will be enrolled from Acupuncture and Moxibustion out-patient or in-patient department of the First affiliated Hospital of Guangzhou university of Chinese medicine. The flow chart is shown in Figure 1. The protocol is composed based on SPIRIT checklist ${ }^{[18]}$ and the Declaration of Helsinki ${ }^{[19]}$. The trial has been approved by the Ethics Committee of the First Affiliated Hospital of Guangzhou University of Chinese Medicine (K[2021]014) and has been registered at the Chinese 
Clinical Registry (ChiCTR2100046604).

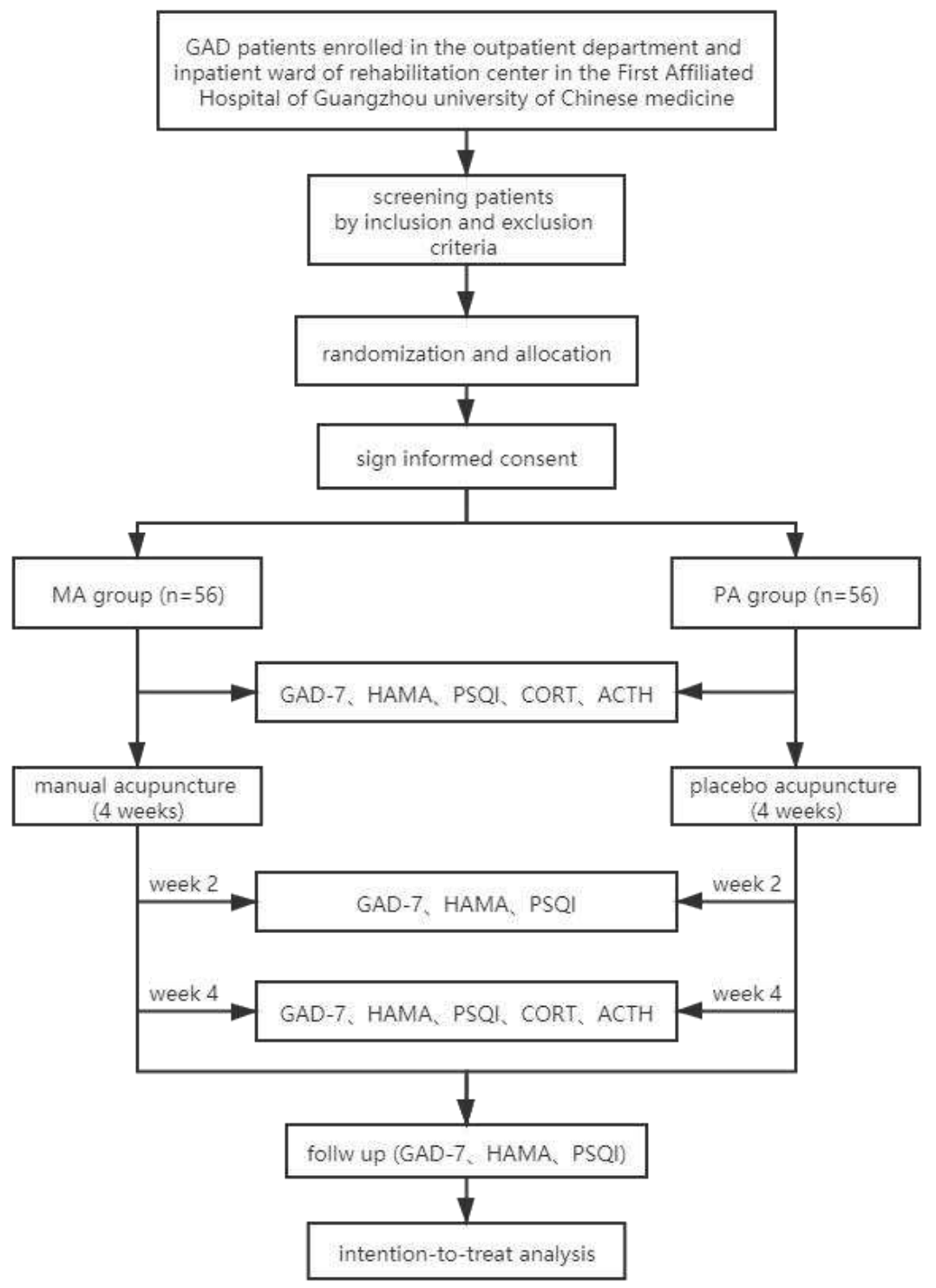

Figure 1 trial flow chart

\section{Sample size}

According to the preliminary trials ${ }^{[20,21]}$, we chose HAMA score as the outcome to calculate the sample size. After treatment, the HAMA score of manual acupuncture was $14.0 \pm 3.2$ and the HAMA score of placebo acupuncture was $19.4 \pm 5.3$. PASS version 15.0 (NCSS, LLC. Kaysville, Utah, USA) was used to estimate the sample size with a power level of $90 \%$ and a two-sided significant level of $5 \%$. After calculating, we set the sample size of 44 patients in each group to observe the statistical difference of two groups. With a $20 \%$ withdrawal rate, we plan to enroll a total of 112 patients with 56 patients in each group.

\section{Randomization and allocation concealment}

According to the random number sequence generated by computer, the patients 
will be randomly divided into either the manual acupuncture (MA) group or the placebo acupuncture (PA) group in a 1:1 ratio. An independent researcher put the card written random number and group assignment into a sealed envelope. The intervenors will allocate enrolled patients into MA group or PA group on the basis of the card in the envelop.

\section{Blinding}

Due to the nature of acupuncture, it's hard to blind patients and intervenor in the same time. To solve this problem, Wang Yuting designed a new placebo acupuncture needle pedestal and had applied for a patent in China (202120398857.9). The new placebo needle can suit for different position of the acupoints and different needling angles (Figure 2). There is a slideway in the base, so the tube can slide over it to change different insert angle. The tube is opaque so that patients cannot see whether the pinpoint of needles insert into skin or not. Therefore, patients are unaware of whether they receive MA or PA. To test the participant-blinding effects, the first two patients will be randomly selected from the study to guess whether they have received MA or PA within 5 minutes after one of the treatment sessions in weeks 2 and 4. Besides, researchers who analyze data are unaware of group assignment either to minimize the potential source of bias.

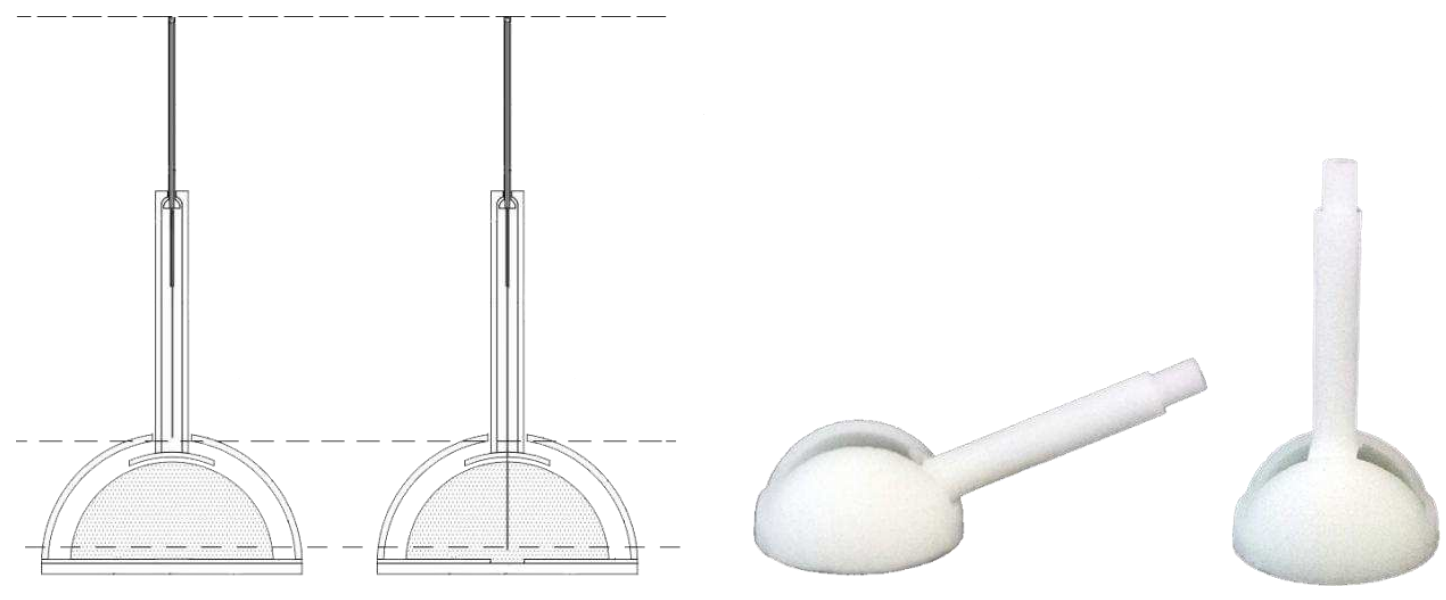

Figure 2 placebo acupuncture needles pedestal

\section{Patient recruitment}

112 GAD patients will be recruited through, but not limited to, reviewing poster pasted in the hospital lobby and wechat moments which can be seen by potential patients. Eligible patient who is meeting the inclusion criteria and exclusion criteria will be enrolled in the outpatient department and inpatient ward of rehabilitation center in the First Affiliated Hospital of Guangzhou university of Chinese medicine.

\section{Inclusion criteria}

1. Aged 45 to 55 years old (female only).

2. Meet the diagnostic criteria of DSM- $5^{[1]}$ for GAD.

3. The score of GAD-7 is over 10 .

4. No anti-anxiety drugs or other psychotropic drugs in the last 2 weeks. 
5. No participation in any other research in the last 1 month.

\section{Exclusion criteria}

1. Diagnosed by other severe illness such as heart failure, tumors, renal failure and so on.

2. Diagnosed by psychotic illness such as schizophrenia, agoraphobia and so on clearly.

3. Addicted to something like alcohol or drugs.

4. Afraid of being needled.

5. Pregnancy or breastfeeding.

\section{Inventions}

All eligible patients will receive 12 sessions of MA or PA (4 weeks, three times a week). Each session will take 30 minutes. The acupuncture therapists have acupuncture education from Guangzhou University of Chinese Medicine (range 1-3 year full-time academic studies) or have clinical experience in the First Affiliated Hospital of Guangzhou University of Chinese Medicine (range 2-5 years). In addition, all intervenors are trained for totally understanding the standard of operation. The acupoints of MA and PA group are the same, including Sishenzhen (one of the main acupoints in Jin's 3 Needle ${ }^{[2]}$ ), Shenting (DU24), Yintang (DU29), Shenmen (HT7), Sanyinjiao (SP6). The acupoints locations are according to the "2006 People's Republic of China National Standard" (GB/T1234-2006) with the exhibition in figure 3 . The acupuncture operation is according to the textbook of the $10^{\text {th }}$ Five-Year plan of the Ministry of Health. 

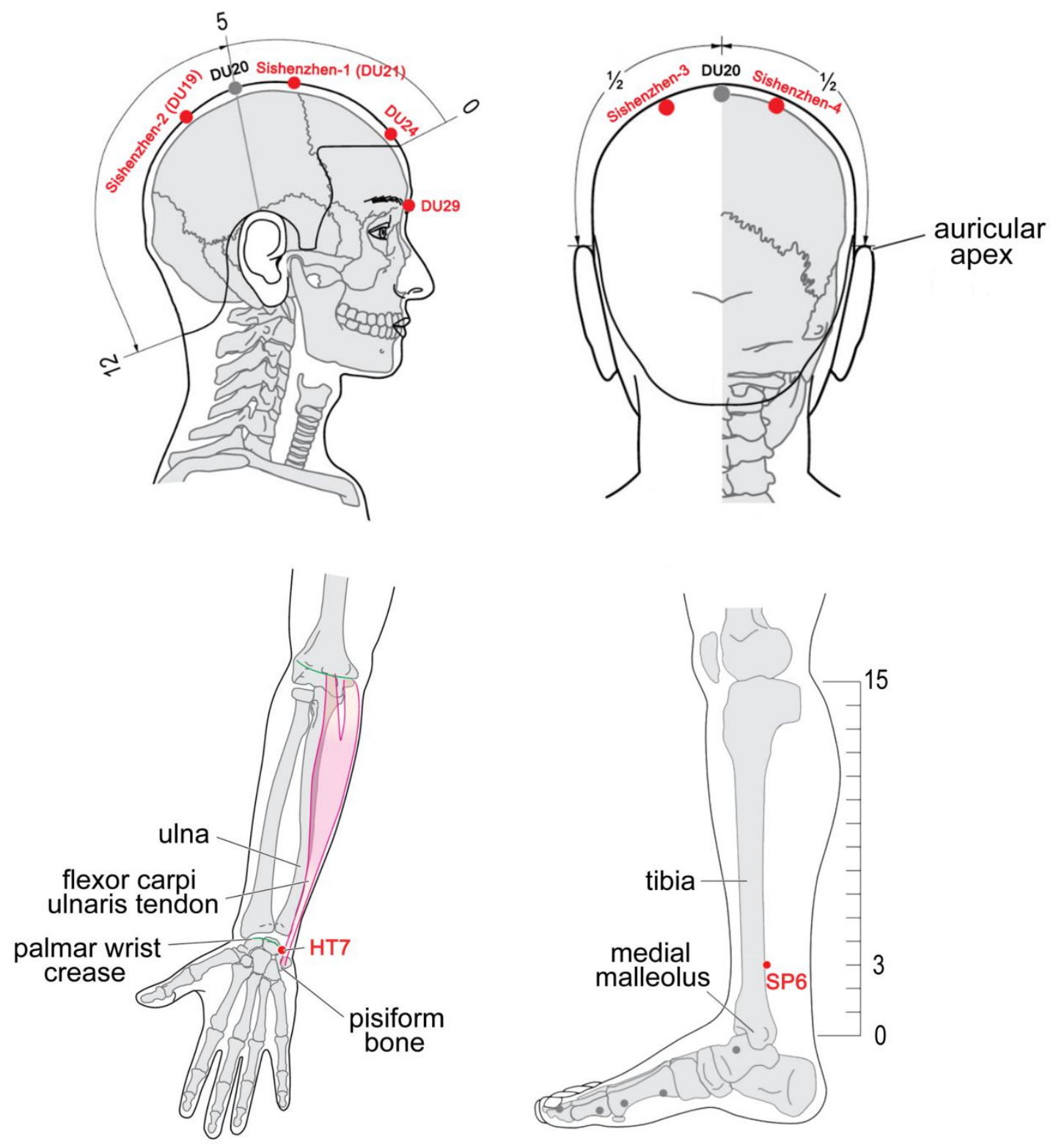

Figure 3 acupoints location

\section{Manual acupuncture (MA) group}

Patients in MA group will be punctured by disposable, stainless steel needles ( $\phi$ $0.30 \mathrm{~mm} \times 40 \mathrm{~mm}$, produced by Huatuo Co. Ltd., China) through our device. The needles will insert into skin and ensure patients have Deqi sensation ${ }^{[23]}$ which is considered as the essential reason for efficacy of acupuncture ${ }^{[24]}$. Depth of puncture is about $25-30 \mathrm{~mm}$. The needles were manipulated three times (at the start, middle and the end of every session) by twirling and lifting until Deqi sensation occurred.

\section{Placebo acupuncture (PA) group}

Patients in PA group will receive placebo acupuncture that the needles would not insert into skin and would not induce Deqi sensation. The blunt tipped placebo needle ( $\$ 0.30 \mathrm{~mm} \times 40 \mathrm{~mm}$, produced by Huatuo Co. Ltd., China) can provide participant-blinding effects with a similar appearance to conventional needles but no 
skin penetration.

\section{Outcome measure}

The schedule of the whole procedure is shown in Figure 4. An independent assessor who doesn't know the group assignment will collect outcome data for analyzing.

\begin{tabular}{|c|c|c|c|c|c|c|c|c|}
\hline \multirow[b]{2}{*}{ Time point } & \multicolumn{8}{|c|}{ Study Period } \\
\hline & Week -1 & Week 0 & Week 1 & Week 2 & Week 3 & Week 4 & Week 8 & Week 16 \\
\hline \multicolumn{9}{|l|}{ Enrollment: } \\
\hline Eligibility screen & $x$ & & & & & & & \\
\hline Informed consent & $x$ & & & & & & & \\
\hline Randomization & & $x$ & & & & & & \\
\hline Allocation & & $x$ & & & & & & \\
\hline \multicolumn{9}{|l|}{ Intervention: } \\
\hline \multicolumn{9}{|l|}{ MA group } \\
\hline \multicolumn{9}{|l|}{ PA group } \\
\hline \multicolumn{9}{|l|}{ Assessments: } \\
\hline GAD-7 & & $x$ & & $x$ & & $x$ & $x$ & $x$ \\
\hline HAMA & & $x$ & & $x$ & & $\times$ & $x$ & $x$ \\
\hline PSQI & & $x$ & & $x$ & & $x$ & $x$ & $x$ \\
\hline $\mathrm{ACTH}$ & & $x$ & & & & $x$ & & \\
\hline Cor & & $x$ & & & & $x$ & & \\
\hline \multicolumn{9}{|l|}{ Safety: } \\
\hline Vital signs & & $x$ & & $x$ & & $x$ & & \\
\hline Adverse events & & & $x$ & $x$ & $x$ & $x$ & & \\
\hline
\end{tabular}

Figure 4 procedure of enrollment, intervention, assessment and safety

\section{Primary outcome}

\section{GAD-7 score}

The primary outcome of this study is the score of GAD-7 scale ${ }^{[25]}$, which measures the severity of GAD particularly. GAD-7 is a 7-item scale, and rated on a 3 -point scale with total score ranges from 0 to 21 . If the score of GAD-7 is 10 or even higher ${ }^{[25]}$, a patient will be diagnosed with GAD. Besides, the score of GAD-7 indicates the level of anxiety. The higher score GAD-7 is, the more anxious patient is. GAD-7 will be assessed before randomization to ensure the diagnosis. If the patient is enrolled, GAD-7 will be assessed in the first day, the $14^{\text {th }}$ day and the $28^{\text {th }}$ day of the trial. 


\section{Secondary outcome}

\section{HAMA score}

The secondary outcome of this study is the score of HAMA scale ${ }^{[26]}$, which measures the severity of anxiety. HAMA is a 14-item scale, and rated on a 4-point scale with total score ranges from 0 to 56. Higher score of HAMA means higher range of anxiety. According to studies, score higher than 28, severe anxiety; score between 28 and 21, moderate anxiety; score between 20 to 14, mild anxiety; score lower than 14 , no anxiety. HAMA will be assessed in the first day, the $14^{\text {th }}$ day and the $28^{\text {th }}$ day of the trial.

\section{PSQI score}

The secondary outcome of this study is the score of PSQI ${ }^{[27]}$, which measures the quality of sleep in the last one month. PSQI is a 19-item questionnaire, divided into 7 parts including sleep quality, sleep latency, sleep duration, habitual sleep efficiency, sleep disturbances, use of sleeping medication, and daytime dysfunction. The score of PSQI is inversely proportional to the quality of sleep. PSQI will be assessed in the first day, the $14^{\text {th }}$ day and the $28^{\text {th }}$ day of the trial.

\section{CORT and ACTH}

It is suggested that anxious subjects demonstrate greater CORT and ACTH concentrations $^{[28]}$. In this study, we will draw the blood samples and investigate the CORT and ACTH before and after acupuncture. The enzyme-linked immunosorbent assay (ELISA) will be used to measure CORT and ACTH. These related tests will be carried out by the clinical laboratory of the First Affiliated Hospital of Guangzhou University of Traditional Chinese Medicine.

\section{Safety outcome}

Researchers will record body temperature, respiratory rate, pulse rate and blood pressure in the first day, the $14^{\text {th }}$ day and the $28^{\text {th }}$ day of the treatment. In addition, researchers should be recorded adverse events (AEs) caused by acupuncture in the case report forms (CRF) truthfully. Besides, intervenors are supposed to treat patients with AEs as soon as possible to comfort patients and eliminate the AEs. After treatment of AEs, researchers will ask patients whether they would like to continue the trial or not and respect their will.

\section{Follow-up}

After the completion of 12-session acupuncture, we will follow patients via telephone or wechat to score GAD-7, HAMA and PSQI in first month and the third month to trace back whether the efficacy can maintain.

\section{Data analysis}

There are two independent study assistants input the data and check by themselves whether inputting data have discrepancies and correct them. IBM SPSS Statistics version 26 (IBM SPSS Inc., Chicago, USA) will be used for analyzing data. 
GAD-7, HAMA and PSQI scores assessed in each group will be performed normality test. If the scores obey normal distribution, one-way analysis of variance will be performed. If not, the Kruskal-Wails rank sum test will be performed. The repeatedly assessed scores of GAD-7, HAMA and PSQI in MA or PA group will be performed normality test. If the scores obey normal distribution, repeated measurement analysis of variance will be performed. If not, the Friedman rank sum test will be performed. CORT and ACTH will be performed covariance analysis.

All statistical tests will be two-sided and significant level will be set at 0.05 . If patient dropped out, we follow the intention-to-treat (ITT) and use the last score or serological indexes assessed as the final score or indexes.

\section{Quality control}

There are two independent study assistants input the data to avoid mistyping. Before the beginning of the trial, all intervenors will be trained, and another assistant will follow the intervention procedures and stay on the outpatient department and inpatient ward to ensure consistency of acupuncture intervention among intervenors. In addition, quality inspectors will check out CRF randomly to make sure if there were record error.

\section{Discussion}

According to recent meta-analysis or systematic reviews ${ }^{[29,30]}$, acupuncture seems to have a better treating efficacy on GAD, but the conclusion is limited. There are many articles compared efficacy between MA and PA, but the conclusions are different. Some reviews ${ }^{[31-34]}$ drew a conclusion that MA definitely better than PA, other review $^{[35]}$ found no difference between MA and PA. The jury is out on whether or not there is existing acupuncture placebo effect. To our best knowledge, no trial study the efficacy of MA versus CBT in treating GAD. In the present research ${ }^{[36] \text {, }}$ CBT combine electro acupuncture (EA) lower HAMA score than CBT or EA alone. In China, CBT is not so general like western countries. And the expense of CBT is much higher than acupuncture, so that a few GAD patients will choose CBT to treat GAD. Besides, there is no licensed psychiatric doctor in our team, it's difficult to carry on CBT. Therefore, this trial compared MA and PA to make sure whether there is placebo effect and provide clinical evidence of placebo effect of acupuncture.

There are many types of anxiety disorders and GAD is one of the most common type. GAD-7 scale was designed for this special type of anxiety disorders. Due to the specificity of GAD-7 and the generalization of HAMA, we use GAD-7 as the primary outcome and HAMA as the secondary outcome to measure the change of patients' anxiety level. We use PSQI as secondary outcome because there is high rate of comorbidity between insomnia and $\mathrm{GAD}^{[37,38]}$. Less of sleeping will aggravate patients' mental burden and worsen their anxiety. Therefore, we want to find whether or not there has connection between GAD and sleeping. Besides, we take blood sample of GAD patients to evaluate the change of CORT and ACTH during the treatment. If the level of CORT and ACTH is associated with level of GAD, acupuncture may treat GAD via HPA axis. 
This study has room for improvement. Firstly, due to the nature of acupuncture, we spare no effort to keep blind, but we still cannot find a perfect way to achieve double blind. We can make pedestal of acupuncture needles look like the same and simulate the feeling of being needled by touched by semi blunt needles. But experienced acupuncturists can feel the difference of needles pierced skin or not. Secondly, we do not set a CBT group and compare the efficacy of CBT and acupuncture in treating GAD. Thirdly, this study is a single center trial, we hope that this study will provide more clinical evidence and design a multicenter trial to confirm the efficacy of acupuncture in treating GAD.

\section{Trial status}

The trial is carrying out right now and the end date of enrollment is expected on May 1,2022.

\section{List of abbreviations}

GAD: Generalized anxiety disorder

GAD-7: Generalized Anxiety Disorder Scale

HAMA: Hamilton Anxiety Scale

PSQI: Pittsburgh sleep quality index

CORT: cortisol

ACTH: adrenocorticotropic hormone

MA: manual acupuncture

PA: placebo acupuncture

ITT: intention-to-treat

SSRIs: Serotonin reuptake inhibitors

CBT: cognitive behavioral therapy

HPA axis: hypothalamic-pituitary-adrenal axis

AEs: adverse events

CRF: case report forms

\section{Authors' contributions}

Lixing Zhuang conceptualized and designed the study. Xin Liu and Xiaoyan Xie drafted the manuscript. Yingjia Li and Meichen Li helped to perform the GAD data collection. Yuting Wang designed the new placebo acupuncture needle pedestal and performed the Clinical Trial Registration. Nanbu Wang was in charge of data analysis. Muxi Liao will help analyse data. Lixing Zhuang provided methodological recommendations and will manage the research. All authors have agreed on the journal to which the article has been submitted; and agree to be accountable for all aspects of the work.

\section{Funding}

This work was supported by the National Administration of Traditional Chinese Medicine (GZY-KJS-2020-072), the Science and Technology Department of Guangdong Province (2018-5) and the Innovative Clinical Research Project of the First Affiliated Hospital of Guangzhou University of Chinese Medicine (2019ZWB07). The funding body had no role in the study design, 
data collection, statistical analysis, or interpretation or preparation of the manuscript.

\section{Ethic approval and consent to participate}

The trial has been approved by the Ethics Committee of the First Affiliated Hospital of Guangzhou University of Chinese Medicine (K[2021]014). Before inclusion in the study, all patients have to provide written informed consent.

\section{Consent for publication}

All authors have agreed on the journal to which the article will be submitted, gave final approval of the version to be published, and agree to be accountable for all aspects of the work.

\section{Competing interests}

The authors declare that they have no conflicts of interest.

\section{Author details}

${ }^{1}$ Clinical Medical College of Acupuncture Moxibustion and Rehabilitation, Guangzhou University of Chinese Medicine, 510000, China.

${ }^{2}$ Department of Rehabilitation, The First Affiliated Hospital of Guangzhou University of Chinese Medicine, 510000, China.

\section{References:}

[1] Pub AP. American Psychiatric Association. Diagnostic and statistical manual of mental disorders (DSM-5 $\left.{ }^{\oplus}\right)[\mathrm{M}] .2013$.

[2] Reed G, First M, Kogan C, et al. Innovations and changes in the ICD-11 classification of mental, behavioural and neurodevelopmental disorders [J]. World psychiatry : official journal of the World Psychiatric Association (WPA), 2019, 18(1): 3-19.

[3] Alonso J, Lépine J. Overview of key data from the European Study of the Epidemiology of Mental Disorders (ESEMeD) [J]. The Journal of clinical psychiatry, 2007, 3-9.

[4] Thibaut F. Anxiety disorders: a review of current literature [J]. Dialogues in clinical neuroscience, 2017, 19(2): 87-88.

[5] Ma X, Xiang $Y$, Cai Z, et al. Generalized anxiety disorder in China: prevalence, sociodemographic correlates, comorbidity, and suicide attempts [J]. Perspectives in psychiatric care, 2009, 45(2): 119-127.

[6] Bandelow B, Michaelis S, Wedekind D. Treatment of anxiety disorders [J]. Dialogues in clinical neuroscience, 2017, 19(2): 93-107.

[7] Arias H, Targowska-Duda K, García-Colunga J, et al. Is the Antidepressant Activity of Selective Serotonin Reuptake Inhibitors Mediated by Nicotinic Acetylcholine Receptors? [J]. Molecules (Basel, Switzerland), 2021, 26(8): 2149.

[8] Warden D, Trivedi M, Wisniewski S, et al. Early adverse events and attrition in selective serotonin reuptake inhibitor treatment: a suicide assessment methodology study report [J]. Journal of clinical psychopharmacology, 2010, 30(3): 259-266.

[9] Hall J, Kellett S, Berrios R, et al. Efficacy of Cognitive Behavioral Therapy for Generalized Anxiety Disorder in Older Adults: Systematic Review, Meta-Analysis, and Meta-Regression [J]. The American journal of geriatric psychiatry : official journal of the American Association for Geriatric 
Psychiatry, 2016, 24(11): 1063-1073.

[10] Linden $M$, Zubraegel D, Baer T, et al. Efficacy of cognitive behaviour therapy in generalized anxiety disorders. Results of a controlled clinical trial (Berlin CBT-GAD Study) [J]. Psychotherapy and psychosomatics, 2005, 74(1): 36-42.

[11] Edinger J, Sampson W. A primary care "friendly" cognitive behavioral insomnia therapy [J]. Sleep, 2003, 26(2): 177-182.

[12] Fiksdal A, Hanlin L, Kuras Y, et al. Associations between symptoms of depression and anxiety and cortisol responses to and recovery from acute stress [J]. Psychoneuroendocrinology, 2019, 102:44-52.

[13] Shan Q, Ke Y, Wang Y. Value of Plasma and Salivary Cortisol Tests in Generalized Anxiety Disorder [J]. Chinese General Practice, 2020, 23(11): 1372-1375.

[14] Vreeburg S, Zitman F, Van Pelt J, et al. Salivary cortisol levels in persons with and without different anxiety disorders [J]. Psychosomatic medicine, 2010, 72(4): 340-347.

[15] De Weerth C. Do bacteria shape our development? Crosstalk between intestinal microbiota and HPA axis [J]. Neuroscience and biobehavioral reviews, 2017, 83:458-471.

[16] Kinlein S, Phillips D, Keller C, et al. Role of corticosterone in altered neurobehavioral responses to acute stress in a model of compromised hypothalamic-pituitary-adrenal axis function [J]. Psychoneuroendocrinology, 2019, 102:248-255.

[17] Fischer S, Cleare A. Cortisol as a predictor of psychological therapy response in anxiety disorders-Systematic review and meta-analysis [J]. Journal of anxiety disorders, 2017, 47:60-68.

[18] Chan A, Tetzlaff J, Altman D, et al. SPIRIT 2013 statement: defining standard protocol items for clinical trials [J]. Annals of internal medicine, 2013, 158(3): 200-207.

[19] Association WM. World Medical Association Declaration of Helsinki: ethical principles for medical research involving human subjects [J]. JAMA, 2013, 310(20): 2191-2194.

[20] Carvalho F, Weires K, Ebling M, et al. Effects of acupuncture on the symptoms of anxiety and depression caused by premenstrual dysphoric disorder [J]. Acupuncture in medicine : journal of the British Medical Acupuncture Society, 2013, 31(4): 358-363.

[21] Dai J, Qin Y, Hao M. Effect of head acupuncture combined with renmai acupoint acupuncture on symptoms and sleep quality of elderly patients with anxiety disorder [J]. Journal of Sichuan of Traditional Chinese Medicine, 2020, 38(11): 184-187.

[22] Yuan Q, Li J, Liu B, et al. Effect of Jin-3-needling therapy on plasma corticosteroid, adrenocorticotrophic hormone and platelet 5-HT levels in patients with generalized anxiety disorder [J]. Chinese journal of integrative medicine, 2007, 13(4): 264-268.

[23] Park H, Park J, Lee $\mathrm{H}$, et al. Does Deqi (needle sensation) exist? [J]. The American journal of Chinese medicine, 2002, 30(1): 45-50.

[24] Chen S, Guo S, Marmori F, et al. Appraisal of the Deqi Concept among Contemporary Chinese Acupuncturists [J]. Evidence-based complementary and alternative medicine : eCAM, 2013, 2013:538476.

[25] Spitzer R, Kroenke K, Williams J, et al. A brief measure for assessing generalized anxiety disorder: the GAD-7 [J]. Archives of internal medicine, 2006, 166(10): 1092-1097.

[26] Hamilton M. The assessment of anxiety states by rating [J]. The British journal of medical psychology, 1959, 32(1): 50-55.

[27] Buysse D, Reynolds C, Monk T, et al. The Pittsburgh Sleep Quality Index: a new instrument for psychiatric practice and research [J]. Psychiatry research, 1989, 28(2): 193-213. 
[28] Bandelow B, Baldwin D, Abelli $M$, et al. Biological markers for anxiety disorders, $O C D$ and PTSD: A consensus statement. Part II: Neurochemistry, neurophysiology and neurocognition [J]. The world journal of biological psychiatry : the official journal of the World Federation of Societies of Biological Psychiatry, 2017, 18(3): 162-214.

[29] Li M, Xing X, Yao L, et al. Acupuncture for treatment of anxiety, an overview of systematic reviews [J]. Complementary therapies in medicine, 2019, 43:247-252.

[30] Yang X, Yang N, Huang F, et al. Effectiveness of acupuncture on anxiety disorder: a systematic review and meta-analysis of randomised controlled trials [J]. Annals of general psychiatry, 2021, 20(1): 9.

[31] Kim J, Kim S, Lee $\mathrm{H}$, et al. Comparing Verum and Sham Acupuncture in Fibromyalgia Syndrome: A Systematic Review and Meta-Analysis [J]. Evidence-based complementary and alternative medicine : eCAM, 2019, 2019:8757685.

[32] Lenoir D, De Pauw R, Van Oosterwijck S, et al. Acupuncture Versus Sham Acupuncture: A Meta-Analysis on Evidence for Longer-term Effects of Acupuncture in Musculoskeletal Disorders [J]. The Clinical journal of pain, 2020, 36(7): 533-549.

[33] Zhang J, He Y, Huang X, et al. The effects of acupuncture versus sham/placebo acupuncture for insomnia: A systematic review and meta-analysis of randomized controlled trials [J]. Complementary therapies in clinical practice, 2020, 41:101253.

[34] Zhong Y, Luo X, Chen Y, et al. Acupuncture versus sham acupuncture for simple obesity: a systematic review and meta-analysis [J]. Postgraduate medical journal, 2020, 96(1134): 221-227.

[35] Xiang Y, He J, Li R. Appropriateness of sham or placebo acupuncture for randomized controlled trials of acupuncture for nonspecific low back pain: a systematic review and meta-analysis [J]. Journal of pain research, 2018, 11:83-94.

[36] Xing J, Wu X, Liu H, et al. Effects of Electroacupuncture Therapy and Cognitive Behavioral Therapy in Chronic Insomnia: A Randomized Controlled Study [J]. Evidence-based complementary and alternative medicine : eCAM, 2020, 2020:5630130.

[37] Ohayon M. Epidemiology of insomnia: what we know and what we still need to learn [J]. Sleep medicine reviews, 2002, 6(2): 97-111.

[38] Alvaro P, Roberts R, Harris J. A Systematic Review Assessing Bidirectionality between Sleep Disturbances, Anxiety, and Depression [J]. Sleep, 2013, 36(7): 1059-1068. 


\section{Supplementary Files}

This is a list of supplementary files associated with this preprint. Click to download.

- SPIRITchecklist.docx 